\title{
The smallest matroids with no large independent flat
}

\author{
Peter Nelson * \\ Department of Combinatorics and Optimization \\ University of Waterloo \\ Ontario, Canada \\ apnelson@uwaterloo.ca
}

\author{
Sergey Norin ${ }^{\dagger}$ \\ Department of Mathematics \\ McGill University \\ Québec, Canada \\ snorin@math.mcgill.ca
}

Submitted: Sep 4, 2019; Accepted: Dec 21, 2020; Published: Feb 12, 2021

(C) The authors. Released under the CC BY-ND license (International 4.0).

\section{Introduction}

Call a set $S$ in a matroid $M$ a claw of $M$ if $S$ is both a flat and an independent set of $M$. A $k$-claw is a claw of size $k$. These objects were introduced by Bonamy et al. [2] and studied by Nelson and Nomoto [3]; both of these papers consider the structure of 3-claw-free binary matroids.

Here we deal with general matroids, and address the simple extremal question of determining the smallest simple rank- $r$ matroids omitting a given claw; we solve this problem and characterize the tight examples.

Theorem 1.8 of [3] shows that, for $r \geqslant 4$, the unique smallest simple rank- $r$ binary matroid with no 3-claw is the direct sum of two binary projective geometries of ranks $\lfloor r / 2\rfloor$ and $\lceil r / 2\rceil$. We show that, perhaps surprisingly, the exact same construction is also extremal for general matroids, and that its natural generalization is still extremal for excluding larger claws. For integers $r \geqslant 1$ and $t \geqslant 1$, let $M_{r, t}$ denote the matroid that is the direct sum of $t$ (possibly empty) binary projective geometries, whose ranks sum to $r$ and pairwise differ by at most 1 . We prove the following, which was conjectured for the special case of binary matroids in [3].

Theorem 1. Let $r, t \geqslant 1$ be integers. If $M$ is a simple rank-r matroid with no $(t+1)$-claw, then $|M| \geqslant\left|M_{r, t}\right|$. If equality holds and $r \geqslant 2 t$, then $M \cong M_{r, t}$.

Note that for $r \leqslant t$, the matroid $M_{r, t}$ is free and therefore the theorem is trivial. For $t<r<2 t$, there is a rather tame family of exceptional tight examples, which we describe

*Supported by an NSERC Discovery Grant and an Early Researcher Award

${ }^{\dagger}$ Supported by an NSERC Discovery Grant 
in Theorem 8. One can also ask a similar question with 'simple' relaxed to 'loopless'. (One must still insist that $M$ is loopless, since any matroid with a loop has no claw.) In this case the answer is much less interesting; the direct sum of $r-t$ parallel pairs and $t$ coloops has $2 r-t$ elements and is the unique smallest loopless rank- $r$ matroid with no $(t+1)$-claw, this is a consequence of Lemma 7 below.

Graph Theory. The study of the structure of 3-claw-free binary matroids in $[2,3]$ was motivated by structural results in graph theory. In the context of these works, the graph-theoretic notions of induced subgraphs, cliques, chromatic number and forests have analogies in the setting of simple binary matroids: cliques are analogous to projective geometries in the sense of being maximal with a given rank, while claws correspond to induced forests. A graph-theoretic analogue of Theorem 1 using this correspondence would characterize graphs on $r$ vertices with minimum number of edges and no induced forests of given size. From the matroidal point of view, the natural measure of the size of a forest is the number of edges, but there seem to exist no direct analogue of Theorem 1 using this measure. Defining the size of a forest as the number of its vertices works much better, as follows.

Let $G_{n, t}$ denote the graph on $n$ vertices which is a disjoint union of $t$ complete subgraphs, whose sizes pairwise differ by at most one. Turán's classical theorem [5] is equivalent to the statement that $|E(G)| \geqslant\left|E\left(G_{n, t}\right)\right|$ for every graph $G$ on $n$ vertices with no stable set of size $t+1$. This observation implies that the following graph-theoretic analogue of Theorem 1 generalizes Turán's theorem for $n \geqslant 3 t$.

Theorem 2. Let $n, t \geqslant 1$ be integers such that $n \geqslant 3 t$. If $G$ is a graph on $n$ vertices having no forest on $2 t+1$ vertices as an induced subgraph, then $|E(G)| \geqslant\left|E\left(G_{n, t}\right)\right|$. If $n \geqslant 4 t$, then the equality holds only if $G$ is isomorphic to $G_{n, t}$.

We give a short proof of Theorem 2, obtained by adapting one of the standard proofs of Turan's theorem, in Section 4.

Triangle-free matroids. The extremal examples in Theorem 1 have many triangles, and our proof techniques analyze triangles closely. It seems plausible that if $M$ is required to be triangle-free, then the sparsest examples, instead of projective geometries, come from binary affine geometries, which are triangle-free and have 2-claws but no 3-claws. (An affine geometry $\mathrm{AG}(r-1,2)$ is obtained from a projective geometry $\mathrm{PG}(r-1,2)$ by deleting a hyperplane.) This leads us to conjecture the following.

Conjecture 3. Let $t, r$ be integers with $t \geqslant 1$ and $t \mid r$. If $M$ is a simple triangle-free matroid with no $(2 t+1)$-claw, then $|M| \geqslant t 2^{r / t-1}$.

This conjectured bound holds with equality when $M$ is the direct sum of $t$ copies of a rank- $(r / t)$ binary affine geometry; these should be the only cases where equality holds. We prove this in the easy case where $t=1$; see Lemma 16.

In what follows, we use the notation of Oxley [4]; flats of a matroid of rank 1 and 2 are points and lines respectively. We additionally write $|M|$ for $E(M)$. A simplification of $M$ is any matroid obtained from $M$ by deleting all loops and all but one element from 
each parallel class. All such matroids are clearly isomorphic; we write $\operatorname{si}(M)$ for a generic matroid isomorphic to a simplification of $M$, and write $\varepsilon(M)$ for $|\operatorname{si}(M)|$, the number of points of $M$. A family $\mathcal{X}$ of sets are skew in a matroid $M$ if $r_{M}(\cup \mathcal{X})=\sum_{X \in \mathcal{X}} r_{M}(X)$, and we say that a set $X$ is skew to a set $Y$ if $\{X, Y\}$ is a skew family: i.e. $r_{M}(X \cup Y)=$ $r_{M}(X)+r_{M}(Y)$.

\section{The Bound}

In this section we give the easy proof of the lower bound in Theorem 1. Our first lemma shows that the property of being $(t+1)$-claw-free is essentially closed under contraction; if $F$ is a $k$-claw of some simplification of $M$, call $F$ a $k$-pseudoclaw of $M$.

Lemma 4. Let $k \geqslant 1$. If $M$ is a simple matroid and $X \subseteq E(M)$, then every $k$-pseudoclaw of $M / X$ is a $k$-claw of $M$.

Proof. Let $M^{\prime}=(M / X) \backslash P$ be a simplification of $M / X$, and suppose that $M^{\prime}$ has a $k$ claw $F$. Since $F$ is independent in $M / X$, it is independent in $M$, and is skew to $X$ in $M$. Since $F$ is a flat of $M / X$, we have $\varnothing=\operatorname{cl}_{M^{\prime}}(F)-F=\operatorname{cl}_{M}(X \cup F)-(F \cup X \cup P) \supseteq$ $\left(\operatorname{cl}_{M}(F)-F\right)-(X \cup P)$, giving $\operatorname{cl}_{M}(F)-F \subseteq X \cup P$.

The sets $\operatorname{cl}_{M}(F)$ and $X$ are skew in $M$, so $\mathrm{cl}_{M}(F)-F \subseteq P$. Suppose that $e \in$ $\left(\mathrm{cl}_{M}(F)-F\right) \cap P$. Then there exists $e^{\prime} \in E\left(M^{\prime}\right)$ parallel to $e$ in $M / X$; since $e^{\prime} \in$ $\operatorname{cl}_{M}(F)-X \subseteq \operatorname{cl}_{M / X}(F)$ we also have $e \in \operatorname{cl}_{M / X}(F)$ and so $e \in \operatorname{cl}_{M^{\prime}}(F)=F$, contrary to the choice of $e$. It follows that $\mathrm{cl}_{M}(F)-F$ intersects neither $X$ nor $P$ so is empty; therefore $F$ is a $k$-claw of $M$.

Let $f(r, t)=\left|M_{r, t}\right|$. Since a rank- $n$ projective geometry has $2^{n}-1$ elements, we clearly have $f(r, t)=(t-a) 2^{\lfloor r / t\rfloor}+a 2^{\lceil r / t\rceil}-t$, where $a \in\{0, \ldots, t-1\}$ is the integer with $a \equiv r$ $(\bmod t)$. More importantly for our purposes, we can define $f$ recursively; it is easy to check that $f(r, t)=r$ for all $0 \leqslant r \leqslant t$ and $f(r, t)=2 f(r-t, t)+t$ for $r>t$. We use this recurrence and the previous lemma to prove the lower bound in our main theorem.

Theorem 5. If $t \geqslant 1$ is an integer and $M$ is a simple rank-r matroid with no $(t+1)$-claw, then $|M| \geqslant f(r, t)$.

Proof. Let $M$ be a counterexample for which $r+|M|$ is minimized. If $M$ is a free matroid then clearly $r \leqslant t$, in which case $f(r, t)=r \leqslant|M|$ so $M$ is not a counterexample. Therefore $M$ has a non-coloop $e$.

Since $|M \backslash e|<|M| \leqslant f(r, t)$ but $M \backslash e$ is not a counterexample, there must be a $(t+1)$-claw $S^{\prime}$ in $M \backslash e$. Now the matroid $M \mid \operatorname{cl}_{M}\left(S^{\prime}\right)$ has rank $t+1$ and has at most $t+2$ elements, so has at most one circuit. There is thus a $t$-element subset $S$ of $\operatorname{cl}_{M}\left(S^{\prime}\right)$ containing at most $|C|-2$ elements of each $C$ circuit of $M$; this set $S$ is a $t$-claw.

If there is some rank- $(t+1)$ flat $F$ containing $S$ for which $|F-S|=1$, then $F$ is a $(t+1)$-claw. Therefore every such flat satisfies $|F-S| \geqslant 2$; since $S$ is a flat, it follows that every parallel class of $M / S$ has size at least 2 . Moreover, $\operatorname{si}(M / S)$ is a rank- $(r-t)$ 
matroid that by Lemma 4 has no $(t+1)$-claw; inductively we have $|\operatorname{si}(M / S)| \geqslant f(r-t, t)$. Now

$$
|M|=|S|+|M / S| \geqslant t+2|\operatorname{si}(M / S)| \geqslant t+2 f(r-t, t)=f(r, t),
$$

as required.

\section{Equality}

We now characterize matroids for which the bound in Theorem 5 holds with equality. This requires two lemmas; the first (which uses Tutte's characterization of binary matroids as those with no $U_{2,4}$-minor [6]) corresponds to the case $t=1$ of Theorem 5 .

Lemma 6. If $M$ is a simple rank-r matroid with no 2-claw, then $|M| \geqslant 2^{r}-1$. If equality holds then $M \cong \mathrm{PG}(r-1,2)$.

Proof. Let $M$ be a minor-minimal counterexample. Clearly $r(M) \geqslant 3$. Let $e \in E(M)$ and let $H$ be a hyperplane of $M$ not containing $e$. Since $M \mid H$ has no 2-claw, we have $|H| \geqslant 2^{r-1}-1$ by the minimality of $M$. For each $x \in H$, the line spanned by $x$ and $e$ contains an element of $E(M)-\{e, x\}$, and these lines pairwise intersect only in $e$, so we see that $|M| \geqslant 2|H|+1 \geqslant 2^{r}-1$ as required.

If $|M|=2^{r}-1$, then equality holds above, so $|H|=2^{r-1}-1$ and thus $M \mid H \cong \mathrm{PG}(r-$ $2,2)$. Moreover, for each $x \in H$ we have $\left|\operatorname{cl}_{M}(\{e, x\})\right|=3$ and $E(M)=\cup_{x \in H}\left(\operatorname{cl}_{M}(\{e, x\})\right)$, which implies that $\operatorname{si}(M / e) \cong M \mid H \cong \mathrm{PG}(r-2,2)$ so $M / e$ is binary. The choice of $e$ was arbitrary, so $M / e$ is binary for all $e$; since $r \geqslant 3$ this gives that $M$ has no $U_{2,4}$-minor so is binary. Since $M$ is simple with $2^{r}-1$ elements, this implies $M \cong \mathrm{PG}(r-1,2)$, a contradiction.

Note that if $t<r<2 t$ then $\left|M_{r, t}\right|=2 r-t$. In this range, the matroids $M_{r, t}$ are not the only ones satisfying the bound in Theorem 5 with equality. The other examples include direct sums of circuits and coloops, and the matroid $M_{r, t}$ is the special case where all these circuits are triangles. The following lemma shows that these are the only examples. It also implies the characterization of the smallest $(t+1)$-claw-free matroids that are not required to be simple that was claimed in the introduction.

Lemma 7. Let $r \geqslant t \geqslant 1$ be integers. If $M$ is a loopless rank-r matroid with no $(t+1)$ claw, then $|M| \geqslant 2 r-t$. If equality holds, then $M$ is the direct sum of $r-t$ circuits and some number of coloops.

Proof. Suppose first that every circuit of $M^{*}$ has at most two elements. Then, since $M^{*}$ is coloopless, it is the direct sum of loops and parallel classes of size at least two; let $\mathcal{P}$ be its set of parallel classes, so $r\left(M^{*}\right)=|\mathcal{P}|$ and $r=|M|-|\mathcal{P}|$. Let $U$ be a set comprising exactly two elements from each $P \in \mathcal{P}$. Since $r\left(M^{*} \mid U\right)=r\left(M^{*}\right)$, the set $(E-U)$ is independent in $M$, and since $M^{*} \mid U$ is coloopless, the set $(E-U)$ is also a flat of $M$, so is a claw of $M$. By hypothesis, it follows that $t \geqslant|E-U|=|M|-2|\mathcal{P}|$. Therefore $2 r-t \leqslant 2(|M|-|\mathcal{P}|)-(|M|-2|\mathcal{P}|)=|M|$, as required. If equality holds, then 
$t=|M|-2|\mathcal{P}|=r-|\mathcal{P}|$, so $|\mathcal{P}|=r-t$. By the definition of $\mathcal{P}$, each $P \in \mathcal{P}$ is a circuit of $M$, and each other element of $M$ is a coloop; this gives the required structure.

We may therefore assume $M^{*}$ has a circuit $C$ of size at least 3 . Let $B$ be a basis of $M^{*}$ containing all but one element of $C$. Since $M^{*}$ has no coloops, for each $x \in B$, there is a circuit $C_{x}$ of $M^{*}$ for which $x \in C_{x}$ and $\left|C_{x} \cap B\right|=\left|C_{x}\right|-1$; choose the $C_{x}$ so that $C_{x}=C$ for each $x \in C$. Let $X=\cup_{x \in X} C_{x}$. Since each $C_{x}$ contains only one element outside $B$ and the element of $C-B$ is chosen at least twice, we have $|X|<2 r\left(M^{*}\right)$.

By construction, the set $X$ contains a basis and, since $X$ is a union of circuits of $M^{*}$, the matroid $M^{*} \mid X$ has no coloops. Let $Y=E(M)-X$; by construction the set $Y$ is independent in $M$, and $M / Y$ has no loops, so $Y$ is a flat, and thus a claw, of $M$. Hence $|Y| \leqslant t$ and so $|X| \geqslant|M|-t$. By our upper bound on $|X|$, this gives $|M|-t<2 r\left(M^{*}\right)=2(|M|-r)$ and so $|M|>2 r-t$, as required.

We are now ready to strengthen Theorem 5 with an equality characterisation. Note that both outcomes in the equality case imply that $M$ has a $t$-claw.

Theorem 8. Let $t \geqslant 1$. If $M$ is a simple rank-r matroid with no $(t+1)$-claw, then $|M| \geqslant f(r, t)$. If equality holds, then either

- $M \cong M_{r, t}$, or

- $t<r<2 t$ and $M$ is the direct sum of coloops and exactly $r-t$ circuits, not all of which are triangles.

Proof. Consider a counterexample $M$ for which $|M|+r$ is minimized. Clearly $r>t$, as otherwise $|M| \geqslant r=f(r, t)$ and there is nothing to prove. Therefore $M$ is not a free matroid, since otherwise any $(t+1)$-element subset of $E(M)$ is a claw.

Claim 9. $M$ has a t-claw.

Subproof: Let $e$ be a non-coloop of $M$; by the minimality of $M$, the matroid $M \backslash e$ has a $(t+1)$-claw $F$; now $M \mid \mathrm{cl}_{M}(F)$ has rank at least $t+1$ and has at most $t+2$ elements, so has at most one circuit. There is thus a $t$-element subset of $\operatorname{cl}_{M}(F)$ that contains at most $|C|-2$ elements of each circuit $C$ of $M \mid \mathrm{cl}_{M}(F)$; this set is a $t$-claw of $M$.

Call a $t$-claw $S$ of $M$ generic if no four-point line of $M$ intersects $S$, and exactly $f(r-t, t)$ triangles of $M$ intersect $S$. Let $S$ be a $t$-claw of $M$, chosen not to be generic if such a choice is possible.

Claim 10. $|M|=f(r, t)$, each parallel class of $M / S$ has size 2 , and $\varepsilon(M / S)=f(r-t, t)$.

Subproof: The matroid $M / S$ has rank $r-t$ and, by Lemma 4, has no $(t+1)$-pseudoclaw. Therefore $\operatorname{si}(M / S)$ has no $(t+1)$-claw, so $\varepsilon(M / S) \geqslant f(r-t, t)$. Moreover, if some parallel class $Y$ of $M / S$ has size 1 , then $S \cup Y$ is a $(t+1)$-claw of $M$, so every parallel class of $M / S$ has size at least 2 , giving $|M / S| \geqslant 2 \varepsilon(M / S)$. Therefore

$$
f(r, t) \geqslant|M| \geqslant 2 \varepsilon(M / S)+|S| \geqslant 2 f(r-t, t)+t=f(r, t) .
$$

Equality holds throughout, which gives the claim. 
The matroid $\operatorname{si}(M / S)$ has no $(t+1)$-claw and has $f(r-t, t)$ elements, so inductively satisfies one of the conclusions of the theorem. For each component $N$ of $M / S$, the matroid $\operatorname{si}(N)$ is either a circuit or a binary projective geometry.

Claim 11. Let $e_{1}, e_{2} \in E(M / S)$. If $e_{1}$ and $e_{2}$ are in different components, then $M / S$ has a t-pseudoclaw containing $e_{1}$ and $e_{2}$. If $e_{1}$ and $e_{2}$ are in the same component, then there is a $(t-1)$-element set $U$ such that $U \cup\left\{e_{1}\right\}$ and $U \cup\left\{e_{2}\right\}$ are both $t$-pseudoclaws of $M / S$.

Subproof: We first argue that $M / S$ has a $t$-pseudoclaw. Since $\operatorname{si}(M / S)$ satisfies one of the outcomes of the theorem, this only fails if $\operatorname{si}(M / S) \cong M_{r-t, t}$ and $r-t<t$. If this holds then $|M|=f(r, t)=2 r-t$ and $M$ satisfies the hypothesis of Lemma 7 , so is the direct sum of coloops and $r-t$ circuits. If these circuits are all triangles then $M \cong M_{r, t}$, and otherwise $M$ satisfies the second outcome of the theorem; both are contrary to the choice of $M$ as a counterexample. Therefore $M / S$ has a $t$-pseudoclaw.

Since every component of $\operatorname{si}(M / S)$ is a circuit or projective geometry, given any pseudoclaw $K$ of $M$ and any $e, e^{\prime}$ in the same component of $M / S$ for which $e \in K$ and $e^{\prime} \notin K$, the set $(K-e) \cup\left\{e^{\prime}\right\}$ is also a pseudoclaw. Since $M / S$ has at least one $t$-pseudoclaw, both conclusions of the claim easily follow.

The above claim implies in particular that every element of $M / S$ is in a $t$-pseudoclaw.

Claim 12. For each t-pseudoclaw $U$ of $M / S$, there is a bijection $\psi_{U}$ from $U$ to $S$ so that for each $e \in U$, the flat $T_{e}=\operatorname{cl}_{M}\left(e, \psi_{U}(e)\right)$ is a triangle of $M$, and so that $M \mid \operatorname{cl}_{M}(S \cup U)=$ $\oplus_{e \in U}\left(M \mid T_{e}\right)$.

Subproof: Since the closure of $U$ in $M / S$ is obtained from $U$ by extending each element of $U$ once in parallel, we have $\left|\operatorname{cl}_{M}(S \cup U)\right|=|S|+2|U|=3 t$. By Lemma 7, it follows that the simple rank-2t matroid $M^{\prime}=M \mid \mathrm{cl}_{M}(S \cup U)$ is the direct sum of $t$ circuits and some set of coloops, and therefore that is precisely the direct sum of $t$ triangles. Since $S$ is a $t$-claw of $M^{\prime}$ and $U$ is a $t$-pseudoclaw of $M^{\prime} / S$, both $S$ and $U$ must be transversals of this set of triangles. The claim follows.

Every element $e$ of $M / S$ is contained in a $t$-pseudoclaw, so the above claim implies that each such $e$ is in exactly one triangle that intersects $S$. Write $\psi(e)$ for the unique element of $S$ for which $e$ and $\psi(e)$ are contained in a triangle; we have $\psi_{U}(e)=\psi(e)$ for each $t$-pseudoclaw $U$ of $M / S$ containing $e$.

Since $S$ is a claw and no rank-1 flat of $M / S$ has more than two elements, no line of $M$ that intersects $S$ has more than three elements. Moreover, each $e \in E(M / S)$ is in exactly one triangle of $M$ that intersects $S$, so the number of triangles of $M$ that intersect $S$ is exactly $\frac{1}{2}|M / S|=\frac{1}{2}(2 \varepsilon(M / S))=f(r-t, t)$. Therefore $S$ is generic. It follows from the choice of $S$ that every $t$-claw of $M$ is generic.

Claim 13. For all $e_{1}, e_{2} \in E(M / S)$, we have $\psi\left(e_{1}\right)=\psi\left(e_{2}\right)$ if and only if $e_{1}$ and $e_{2}$ are in the same component of $M / S$. Moreover, $M / S$ has exactly $t$ components. 
Subproof: Suppose that $e_{1}$ and $e_{2}$ are in the same component of $M / S$. By Claim 11, there is a set $U \subseteq E(M / S)$ such that $U \cup\left\{e_{1}\right\}$ and $U \cup\left\{e_{2}\right\}$ are both $t$-pseudoclaws of $M / S$. For each $i \in\{1,2\}$, there is a bijection $\psi_{i}=\psi_{U \cup\left\{e_{i}\right\}}$ from $U \cup\left\{e_{i}\right\}$ to $S$, and moreover for each $e \in U$ we have $\psi_{1}(e)=\psi(e)=\psi_{2}(e)$. Therefore $\psi_{1}$ and $\psi_{2}$ agree on all $t-1$ elements of $U$; thus $\psi_{1}\left(e_{1}\right)=\psi_{1}\left(e_{2}\right)$ and so $\psi\left(e_{1}\right)=\psi\left(e_{2}\right)$.

Suppose now that $e_{1}$ and $e_{2}$ are in different components of $M / S$. By Claim 11 there is a $t$-pseudoclaw $U$ containing $e_{1}$ and $e_{2}$. Since $\psi_{U}$ is a bijection we have $\psi\left(e_{1}\right)=\psi_{U}\left(e_{1}\right) \neq$ $\psi_{U}\left(e_{2}\right)=\psi\left(e_{2}\right)$, as required.

It follows from the first part that the image of $\psi$ has size equal to the number of components of $M / S$. But clearly the image of $\psi$ contains the image of $\psi_{U}$, which is equal to $S$, for each $t$-pseudoclaw $U$. Therefore $\psi$ has image $S$, so $M / S$ has exactly $|S|=t$ components.

Let $\mathcal{N}$ be the set of components of $M / S$. By Claim 13, for each $N \in \mathcal{N}$ there is some $\psi(N)$ for which $\psi(e)=\psi(N)$ for each $e \in E(N)$. Since $|S|=|\mathcal{N}|=t$, the $t$-tuple $\psi(N): N \in \mathcal{N}$ is a permutation of $S$. For each $N \in \mathcal{N}$, let $\widehat{N}=M \mid(E(N) \cup \psi(N))$.

Claim 14. If $N \in \mathcal{N}$ and $L$ is a line of $M$ intersecting $E(N)$, then either $|L|=2$, or $|L|=3$ and $L \subseteq E(\widehat{N})$.

Subproof: Let $U$ be a $t$-pseudoclaw of $M / S$ containing an element $e \in E(N) \cap L$. Note that $U$ is a generic $t$-claw in $M$, which gives $|L| \leqslant 3$.

Suppose that $|L|=3$. Note that $1=r_{M / S}(e) \leqslant r_{M / S}(L-S) \leqslant r_{M}(L)=2$. If $r_{M / S}(L-S)=2$ then $L$ is a triangle of $M / S$ that intersects the component $N$ of $M / S$, so obviously $L \subseteq E(N)$. If $r_{M / S}(L-S)=1$ then $L \subseteq \operatorname{cl}_{M}(S \cup\{e\}) \subseteq \operatorname{cl}_{M}(S \cup U)$, so 12 gives $L=\mathrm{cl}_{M}(\{e, \psi(e)\})$. Since $L-\psi(e)$ is a two-element rank-1 set in $M / S$, the third element of $L$ is the element of $N$ parallel to $e$, so $L \subseteq E(\widehat{N})$ as required.

For each $N \in \mathcal{N}$ and $e \in E(N)$, let $\tau(e)$ be the number of 3-element lines of $M$ containing $e$, and let $\sigma(e)$ be the number of elements of $E(\widehat{N} \backslash e)$ that are not in a 3element line of $M$ with $e$. By the previous claim we have $2 \tau(e)+\sigma(e)=|\widehat{N} \backslash e|=|N|$.

Claim 15. For each $N \in \mathcal{N}$, every line of $E(\widehat{N})$ has size 3 .

Subproof: Suppose not, so there is some $e \in E(N)$ for which $\sigma(e)>0$. Let $U$ be a $t$ pseudoclaw of $M / S$ containing $e$. Since $U$ is a generic $t$-claw of $M$, we have $\sum_{u \in U} \tau(u)=$ $f(r-t, t)$, so

$$
\begin{aligned}
|M| & =|S|+\sum_{N \in \mathcal{N}}|N| \\
& =t+\sum_{u \in U}(2 \tau(u)+\sigma(u)) \\
& =t+2 f(r-t, t)+\sum_{u \in U} \sigma(u) \\
& \geqslant f(r, t)+\sigma(e) .
\end{aligned}
$$


Since $|M|=f(r, t)$ and $\sigma(e)>0$, this is a contradiction.

Let $N \in \mathcal{N}$. It is clear, since $N$ is obtained from $\widehat{N} / \psi(N)$ by $t-1$ successive extensioncontraction operations, that $r(N) \leqslant r(\widehat{N})-1$. The matroid $\operatorname{si}(N)$ is a circuit or a binary projective geometry, so

$$
|\widehat{N}|=|N|+1 \leqslant 2\left(2^{r(N)}-1\right)+1=2^{r(N)+1}-1 \leqslant 2^{r(\widehat{N})}-1 .
$$

By Claim 15 and Lemma 6 we have $|\widehat{N}| \geqslant 2^{r(\widehat{N})}-1$, so equality holds and therefore each matroid $\widehat{N}$ is a binary projective geometry of rank $r(N)+1$. The sets $E(\widehat{N}): N \in \mathcal{N}$ partition $E(M)$, so

$$
r \leqslant \sum_{N \in \mathcal{N}} r(\widehat{N})=\sum_{N \in \mathcal{N}}(r(N)+1)=r(M / S)+|\mathcal{N}|=t+r(M / S)=r,
$$

so equality holds throughout, and the sets $\{E(\widehat{N}): N \in \mathcal{N}\}$ are skew in $M$. Thus $M$ is the direct sum of $t$ nonempty binary projective geometries. If $M$ has components of ranks $r_{1}, r_{2}$ with $r_{2} \geqslant r_{1}+2$, then deleting both and replacing them with projective geometries of rank $r_{2}-1$ and $r_{1}+1$ respectively gives a matroid $M^{\prime}$ with no $(t+1)$-claw satisfying

$$
|M|-\left|M^{\prime}\right|=2^{r_{2}}+2^{r_{1}}-2^{r_{2}-1}-2^{r_{1}+1}=2^{r_{2}-1}-2^{r_{1}+1}>0,
$$

which contradicts the minimality of $|M|$. It follows that no two components of $M$ have ranks differing by more than 1 , so $M \cong M_{r, t}$, contrary to the choice of $M$ as a counterexample.

Finally, we prove the $t=1$ case of Conjecture 3 as promised.

Lemma 16. If $M$ is a simple rank-r triangle-free matroid with no 3-claw, then $|M| \geqslant$ $2^{r-1}$. If equality holds, then $M \cong A G(r-1,2)$.

Proof. We may assume that $r \geqslant 3$. We first show that every triple of distinct elements of $M$ is contained in a four-element circuit; indeed, given such a triple $I$, since $I$ is not a triangle or a 3-claw, we have $r_{M}(I)=3$ and $\operatorname{cl}_{M}(I) \neq I$. Thus there is some $x \in \operatorname{cl}_{M}(I)-I$. Since $M$ is triangle-free, no pair of elements of $I$ spans $x$, so $I \cup\{x\}$ is a 4-element circuit.

Let $e \in E(M)$. Since $M$ is triangle-free, the matroid $M / e$ is simple. If $M / e$ has a 2-claw $I$, then $I \cup\{e\}$ is clearly a 3-claw of $M$; therefore $M / e$ is 2-claw-free and so $|M / e| \geqslant 2^{r-1}-1$ by Lemma 6 . It follows that $|M| \geqslant 2^{r-1}$ as required.

If equality holds, then $M / e \cong \mathrm{PG}(r-2,2)$ so $M / e$ is binary. This holds for arbitrary $e \in E(M)$; it follows (since $r \geqslant 3$ ) that $M$ has no $U_{2,4}$-minor so is also binary. A simple rank- $r$ triangle-free binary matroid has at most $2^{r-1}$ elements and equality holds only for binary affine geometries (see [1], for example); therefore $M \cong \mathrm{AG}(r-1,2)$. 


\section{Graphs}

Let $g: \mathbb{N} \times \mathbb{N} \rightarrow \mathbb{N}$ be defined recursively by

- $g(n, t)=0$ for $n<2 t$,

- $g(n, t)=3(n-2 t)$ for $2 t \leqslant n \leqslant 4 t$,

- $g(n, t)=g(n-1, t)+\left\lceil\frac{n}{t}\right\rceil-1$, for $n>4 t$.

It is easy to check that $\left|E\left(G_{n, t}\right)\right|=g(n, t)$ for $n \geqslant 3 t$ (although not for smaller $n$ ). The recursion for $n>4 t$ in fact also holds when $3 t<n \leqslant 4 t$. Thus the next theorem implies Theorem 2.

Theorem 17. Let $n, t \geqslant 1$ be integers. Let $G$ be a simple graph on $n$ vertices such that no forest on $2 t+1$ vertices is an induced subgraph of $G$. Then

$$
|E(G)| \geqslant g(n, t)
$$

If equality holds and $n<4 t$, then every component of $G$ is a complete graph on 1,3 or 4 vertices. If equality holds and $n \geqslant 4 t$, then $G$ is isomorphic to $G_{n, t}$.

Proof. We prove the theorem by induction on $|V(G)|$. We may clearly assume that $n \geqslant 2 t+1$, as otherwise the result is easy. Let $v$ be a vertex of $G$ of maximum degree.

If $\operatorname{deg}(v) \leqslant 2$, every component of $G$ is a path or a cycle. Let $S$ be the set of vertices of cycles of $G$, and $b$ be the number of cycles of $G$; note that $b \leqslant \frac{1}{3}|S| \leqslant \frac{1}{3} n$. Clearly $G$ contains an induced forest on $n-b$ vertices, so $n-b \leqslant 2 t$. This gives $n \leqslant 2 t+\frac{n}{3}$, so $n \leqslant 3 t$, which in turn implies that $g(n, t)=3(n-2 t) \leqslant 3 b$. On the other hand, we have $|E(G[S])|=|S|$, so

$$
|E(G)| \geqslant|E(G[S])|=|S| \geqslant 3 b \geqslant g(n, t),
$$

giving the bound. If equality holds, then $E(G)=E(G[S])$ and $b=\frac{1}{3}|S|$, so every component of $G$ is an isolated vertex or triangle. We have argued that $n \leqslant 3 t$; thus $G$ has the claimed structure. We may therefore assume that $\operatorname{deg}(v) \geqslant 3$.

Let $X \subseteq V(G)$ be maximal so that $G[X]$ is a forest, so $|X| \leqslant 2 t$. Let $Z$ be the set of non-isolated vertices of $G[X]$. As $G[X \cup\{w\}]$ contains a cycle for every $w \in V(G) \backslash X$, every such $w$ has at least two neighbors in $Z$. Thus

$$
\sum_{z \in Z} \operatorname{deg}(z) \geqslant|Z|+2|V(G)-X| \geqslant|Z|+2(n-2 t)
$$

Hence there exists $z_{0} \in Z$ such that $\operatorname{deg}\left(z_{0}\right) \geqslant 2(n-2 t) /|Z|+1 \geqslant(n-2 t) / t+1=n / t-1$; thus $\operatorname{deg}(v) \geqslant\left\lceil\frac{n}{t}\right\rceil-1$ by the choice of $v$.

By the above, we can assume that $\operatorname{deg}(v) \geqslant \max \left(3,\left\lceil\frac{n}{t}\right\rceil-1\right)$. Let $H=G-v$. It follows that

$$
|E(G)|=|E(H)|+\operatorname{deg}(v) \geqslant g(n-1, t)+\max \left(3,\left\lceil\frac{n}{t}\right\rceil-1\right)=g(n, t) ;
$$


the last equality is easy to check. This gives the desired bound.

Suppose now that $|E(G)|=g(n, t)$. Then $\operatorname{deg}(v)=\max \left(3,\left\lceil\frac{n}{t}\right\rceil-1\right)$, and $|E(H)|=$ $g(n-1, t)$. Call a component of $H$ with at least two vertices big. By the induction hypothesis, every component of $H$ is a complete graph. Therefore, $H[X]$ is a maximal induced forest in $H$ if and only if $X$ contains at least one vertex from every component of $H$ and exactly two vertices from every big component. It follows that $|X|=2 t$ for every such $X$. (Otherwise we could remove any edge from $H$ to get a graph containing no $(2 t+1)$-vertex induced forest and with fewer than $g(n-1, t)$ edges.)

If each big component of $H$ contains a non-neighbour of $v$, then we can choose a set $X$ as above so that $X \cup\{v\}$ induces a forest on $2 t+1$ vertices, a contradiction. Therefore $H$ has a big component $C$ such that $v$ is complete to $C$. By the induction hypothesis, each big component of $H$ has at least $\max \left(3,\left\lceil\frac{n}{t}\right\rceil-1\right)=\operatorname{deg}(v)$ vertices; it follows that $|V(C)|=\operatorname{deg}(v)=\max \left(3,\left\lceil\frac{n}{t}\right\rceil-1\right)$, and that $G$ is obtained from $H$ by adding a new vertex with neighbourhood $V(C)$.

If $2 t+1 \leqslant n \leqslant 4 t$, then $|V(C)|=\operatorname{deg}(v)=\max \left(3,\left\lceil\frac{n}{t}\right\rceil-1\right)=3$, so $G$ is obtained from $H$ by adding a vertex complete to a component on three vertices; thus, every component of $G$ is complete with 1, 3 or 4 vertices. If $n<4 t$ then this implies that $G$ has the claimed structure. If $n=4 t$ then $|E(G)|=g(4 t, t)=6 t=\frac{3}{2}|V(G)|$ and $G$ has maximum degree 3 . This implies that every vertex of $G$ has degree three, and so $G$ is isomorphic to $G_{4 t, t}$, as required. If $n>4 t$, then $H$ is isomorphic to $G_{n-1, t}$, so $|V(C)|=\operatorname{deg}(v)=\left\lceil\frac{n}{t}\right\rceil-1=\left\lfloor\frac{n-1}{t}\right\rfloor$. Thus $C$ is a smallest component of $H$, so $G$ is isomorphic to $G_{n, t}$, as required.

\section{Acknowledgements}

We thank the anonymous referee for their careful reading and useful comments, and for generously prompting us to determine where equality holds in the last section.

\section{References}

[1] R. C. Bose, R. C. Burton. A characterization of flat spaces in a finite geometry and the uniqueness of the Hamming and the MacDonald codes. J. Combin. Theory 1, 96-104, 1966.

[2] M. Bonamy, F. Kardoš, T. Kelly, P. Nelson, L. Postle. The structure of binary matroids with no induced claw or Fano plane restriction. Advances in Combinatorics. 10.19086/aic.10256, 2019.

[3] P. Nelson, K. Nomoto. The structure of claw-free binary matroids. arXiv: 1807.11543

[4] J. G. Oxley. Matroid Theory (second edition). Oxford University Press, New York, 2011.

[5] P. Turán. On an extremal problem in graph theory. Matematikai és Fizikai Lapok 436-452, 1941.

[6] W.T. Tutte. Lectures on matroids. Journal of Research of the National Bureau of Standards, 1-47, 1965. 